\title{
AUTOMORPHISMS OF THE TOEPLITZ ALGEBRA
}

\author{
PAUL S. MUHLY AND JINGBO XIA
}

(Communicated by Palle E. T. Jorgensen)

\begin{abstract}
We investigate conditions under which a homeomorphism of the unit circle induces, via composition, an automorphism of the $C^{*}$-algebra generated by all the Toeplitz operators with bounded measurable symbols.
\end{abstract}

\section{INTRODUCTION}

Let $L^{2}$ and $H^{2}$ denote, respectively, the Lebesgue space and the Hardy space of the unit circle $\mathbf{T}$. For $f \in L^{\infty}=L^{\infty}(\mathbf{T})$, let $T_{f}$ denote the Toeplitz operator $P M_{f} \mid H^{2}$, where $P: L^{2} \rightarrow H^{2}$ is the orthogonal projection. All the properties of Toeplitz operators stated below without proof can be found in [4]. Given a subalgebra $\mathscr{A}$ of $L^{\infty}$, let $\mathscr{T}(\mathscr{A})$ denote the norm closed algebra generated by all $T_{f}, f \in \mathscr{A}$. In this note we begin an investigation into the structure of the automorphisms of $\mathscr{T}(\mathscr{A})$, concentrating primarily on the case when $\mathscr{A}=L^{\infty}$.

Recall that when $\mathscr{A}=C(\mathbf{T})=C$, the collection of continuous functions on $\mathbf{T}$, one has a short exact sequence

$$
0 \longrightarrow \mathscr{K} \rightarrow \mathscr{T}(C) \stackrel{\lrcorner}{\longrightarrow} C \longrightarrow 0,
$$

where $\mathscr{K}$ is the commutator ideal of $\mathscr{T}(C)$, which coincides with the collection of compact operators on $H^{2}$, and $s$ is the symbol map $s\left(T_{f}\right)=f$. As a result, any automorphism of $\mathscr{T}(C)$ must induce an automorphism of $\mathscr{R}$ and so is implemented by a unitary transformation on $H^{2}$. In addition, because $\mathscr{K}$ is irreducible, the unitary is unique up to a scalar multiple of modulus one. More generally, it follows that if $\mathscr{A}$ contains $C$ then any automorphism of $\mathscr{T}(\mathscr{A})$ must be implemented by an essentially unique unitary transformation.

Suppose that $\varphi$ is an automorphism of the $C^{*}$-algebra $\mathscr{T}(C)$, and let $V$ be the essentially unique operator on $H^{2}$ such that $\varphi(A)=V^{*} A V$ for all $A \in \mathscr{T}(C)$. Then from equation (1.1) $\varphi$ induces a map $\hat{\varphi}: \mathscr{T}(C) / \mathscr{K} \rightarrow$ $\mathscr{T}(C) / \mathscr{K}(\cong C)$ that is an automorphism of $C$. Consequently, there is a homeomorphism $\sigma: \mathbf{T} \rightarrow \mathbf{T}$ such that $\hat{\sigma}(f)=f \circ \sigma$ for all $f \in C$. From index considerations, it is easy to see that such a $\sigma$ must be orientation preserving

Received by the editors April 20, 1991.

1991 Mathematics Subject Classification. Primary 47B35, 47C15; Secondary 46L40.

Research supported in part by grants from the National Science Foundation. 
in the sense that $[\sigma]=[\chi]$ in the fundamental group $\pi_{1}(\mathbf{T})$, where $\chi$ is the identity function $\chi(\tau)=\tau$. For any $f \in C$, then, we have $\varphi\left(T_{f}\right)=T_{f \circ \sigma}+K$ with some $K$ in $\mathscr{K}$ that depends on $\varphi$. On the other hand, by the BDF theory, given any orientation-preserving homeomorphism $\sigma$ of $\mathbf{T}$, there is a compact operator $K$ on $H^{2}$ such that $T_{\sigma}+K=T_{\chi \circ \sigma}+K$ is a unilateral shift. (Also see [4].) Hence there is a unitary operator $U_{\sigma}$ such that $U_{\sigma} T_{\chi} U_{\sigma}^{*}=T_{\sigma}+K$. Thus $\varphi_{\sigma}(A)=U_{\sigma} A U_{\sigma}^{*}$ defines an automorphism of $\mathscr{T}(C)$ such that $\hat{\varphi}_{\sigma}(f)=f \circ \sigma$ for all $f \in C$. In other words, for any orientation-preserving $\sigma$, there is an automorphism $\varphi_{\sigma}$ of $\mathscr{T}(C)$ such that $\varphi_{\sigma}\left(T_{f}\right)=T_{f \circ \sigma}+$ compact for all $f \in C$. Given the BDF theory, the main reason that one can obtain a surjective map from $\operatorname{Aut}(\mathscr{T}(C))$ onto $\operatorname{Aut}(C)$ is that $\mathscr{T}(C)$, as a $C^{*}$-algebra, has a single generator.

When we replace $C$ with $L^{\infty}$ we obtain, in analogy with (1.1), the short exact sequence

$$
0 \longrightarrow \mathscr{C} \rightarrow \mathscr{T}\left(L^{\infty}\right) \stackrel{\circ}{\longrightarrow} L^{\infty} \longrightarrow 0
$$

where $\mathscr{C}$ is the commutator ideal of $\mathscr{T}\left(L^{\infty}\right)$ and $s$ is the symbol map $s\left(T_{f}\right)=$ $f$. Unlike the case of $C$, however, the commutator ideal here, $\mathscr{C}$, is very complex and poorly understood. The most we can say is that it contains $\mathscr{K}$. If $\varphi$ is an automorphism of $\mathscr{T}\left(L^{\infty}\right)$ then we find, as before, that it is spatially implemented by an essentially unique unitary transformation on $H^{2}$ and $\varphi$ induces an automorphism $\hat{\varphi}$ of $\mathscr{T}\left(L^{\infty}\right) / \mathscr{C} \cong L^{\infty}$. As a result, there is a Borel map $\sigma$ of $T$ that preserves Lebesgue null sets (we call such a map a nonsingular measurable transformation) and there is a unitary transformation $U_{\sigma}$ on $H^{2}$ such that

$$
\varphi\left(T_{f}\right)=U_{\sigma}^{*} T_{f} U_{\sigma}=T_{f \circ \sigma}+C,
$$

for some $C$ in $\mathscr{C}$ depending on $\varphi$. At this point, it is natural to ask for a converse. That is, what conditions must one place on a measurable map $\sigma$ in order for it to induce an automorphism of $\mathscr{T}\left(L^{\infty}\right)$ ? Moreover, one is also naturally led to inquire about the relation between $\sigma$ and $U_{\sigma}$, given that $\sigma$ induces an automorphism $\mathscr{T}\left(L^{\infty}\right)$. Is there a sense in which $U_{\sigma}$ is implemented by $\sigma$ ? Note that this second question is interesting even for automorphisms of $\mathscr{T}(C)$ because orientation-preserving homeomorphisms need not be nonsingular measurable transformations in the sense in which we are using the term.

Observe that if $\sigma$ is a nonsingular measurable transformation on $\mathbf{T}$ then there is a unitary operator $V_{\sigma}$ on $L^{2}$ that implements the automorphism of $L^{\infty}$ determined by $\sigma$. It is given by the formula $V_{\sigma} g=g \circ \sigma J^{1 / 2}$ where $J$ is the Radon-Nikodym derivative of the measure $m \circ \sigma$ with respect to Lebesgue measure $m$. The function $J$ is a positive function in $L^{1}$, but little more can be said of it. A little reflection reveals that whether or not $\sigma$ implements an automorphism of $\mathscr{T}\left(L^{\infty}\right)$ involves the structure of the commutator $\left[V_{\sigma}, P\right]$. In order to make any headway with this, one must make some restrictive hypotheses on $\sigma$. In particular, it is natural to assume that $J$ is bounded and this, in turn, forces $\sigma$ to be an absolutely continuous homeomorphism satisfying a Lipschitz condition. We seem to require more, still, and we are not sure what the correct level of generality of our results are. In any case, we shall find in the next section that if $\sigma$ is a $C^{1}$, orientation-preserving homeomorphism with derivative satisfying a Lipschitz condition, then $\sigma$ induces an automorphism of 
$\mathscr{T}\left(L^{\infty}\right)$ and the implementing unitary can be expressed in terms of $\sigma$. Along the way, we shall encounter some new results about composition operators and commutators.

In concluding this introduction, we want to emphasize that it seems to us that the reason it is not clear a priori that an orientation-preserving, nonsingular homeomorphism induces an automorphism of $\mathscr{T}\left(L^{\infty}\right)$ is that unlike $\mathscr{T}(C)$, this algebra is not singly generated and, consequently, BDF cannot be appliedat least not directly.

\section{COMPOSITION OPERATORS AND COMMUTATORS}

In what follows, we will consider homeomorphisms $\sigma$ of $\mathbf{T}$ satisfying the following conditions:

(2.1) $\sigma$ and its inverse $\sigma^{-1}$, as functions on $\mathbf{T}$, are both $C^{1}$;

(2.2) there are an $M>0$ and a $\delta>0$ such that

$$
\left|\frac{d \sigma}{d t}\left(\tau_{1}\right)-\frac{d \sigma}{d \tau}\left(\tau_{2}\right)\right| \leq M\left|\tau_{1}-\tau_{2}\right|^{\delta}
$$

for all $\tau_{1}, \tau_{2} \in \mathbf{T}$;

(2.3) $\sigma$ is orientation-preserving.

If $\sigma$ is a homeomorphism satisfying condition (2.1), then both $d \sigma(\tau) / d \tau$ and $d \sigma^{-1}(\tau) / d \tau$ are bounded away from 0 . Therefore, the composition operators $C_{\sigma} g=g \circ \sigma$ and $C_{\sigma^{-1}} g=g \circ \sigma^{-1}=C_{\sigma}^{-1} g$ are bounded on $L^{2}$.

Let $H$ be the Hilbert transform on $L^{2}$, i.e., $(H g)(\tau)=\sum_{n=-\infty}^{\infty} \operatorname{sgn}(n) a_{n} \tau^{n}$ for $g(\tau)=\sum_{n=-\infty}^{\infty} a_{n} \tau^{n}$ in $L^{2}$. Alternatively, $H$ is given by the singular integral

$$
(H g)\left(e^{i s}\right)=\lim _{\varepsilon \downarrow 0} \frac{1}{2 \pi i} \int_{-\pi}^{\pi} \chi_{\varepsilon}(t-s) \frac{g\left(e^{i t}\right)}{e^{i t}-e^{i s}} d e^{i t}=\frac{1}{2 \pi i} \mathrm{P} . \mathrm{V} . \int_{-\pi}^{\pi} \frac{g\left(e^{i t}\right)}{e^{i t}-e^{i s}} d e^{i t},
$$

where $\chi_{\varepsilon}$ is the characteristic function of $\mathbb{R} \backslash[-\varepsilon, \varepsilon]$.

The following is the key result about the compactness of $\left[C_{\sigma}, H\right]$ and its consequences. We suspect that the hypotheses (2.1)-(2.3) can be weakened somewhat, but we are not sure how.

Theorem 2.1. Let $\sigma$ be a homeomorphism of $\mathbf{T}$ satisfying (2.1), (2.2), and (2.3). Then $H-C_{\sigma^{-1}} H C_{\sigma}$ is a compact operator on $L^{2}$. Furthermore, $P C_{\sigma} P$ is an invertible operator on $H^{2}$.

We postpone the proof for a few minutes. Let

$$
P C_{\sigma} P=V_{\sigma}\left[\left(P C_{\sigma} P\right)^{*}\left(P C_{\sigma} P\right)\right]^{1 / 2}
$$

be the polar decomposition. Then $V_{\sigma}$ is a unitary operator on $H^{2}$.

Theorem 2.2. Let $\sigma$ be a homeomorphism of $\mathbf{T}$ satisfying (2.1), (2.2), and (2.3). Then $V_{\sigma} T_{f} V_{\sigma}^{*}-T_{f \circ \sigma} \in \mathscr{K}$ for all $f \in L^{\infty}$. Thus there is an automorphism $\varphi_{\sigma}$ of $\mathscr{T}\left(L^{\infty}\right)$ such that

$$
\varphi_{\sigma}\left(T_{f}\right)=V_{\sigma} T_{f} V_{\sigma}^{*}=T_{f \circ \sigma}+\text { compact }
$$

for all $f \in L^{\infty}$.

Remark. Observe that the conclusion of Theorem 2.2 is somewhat stronger than expected. It is natural to ask if relaxation of the hypotheses on $\sigma$ may lead to a 
transformation $V_{\sigma}$ such that $V_{\sigma} T_{f} V_{\sigma}^{*}=T_{f \circ \sigma}+C$, where $C$ is only an element in the commutator ideal $\mathscr{C}$.

Proof of Theorem 2.2 given Theorem 2.1. Since $C_{\sigma}^{*}=M_{\mu} C_{\sigma^{-1}}$ with $\mu(\tau)=$ $d m(\sigma(\tau)) / d m(\tau)$, where $m$ is the normalized Lebesgue measure on $\mathbf{T}$, we have

$$
\begin{aligned}
A & :=\left(P C_{\sigma} P\right)^{*}\left(P C_{\sigma} P\right)=P M_{\mu} C_{\sigma^{-1}} P C_{\sigma} P \\
& =P M_{\mu} C_{\sigma^{-1}} C_{\sigma} P-P M_{\mu} C_{\sigma^{-1}}(1-P) C_{\sigma} P=T_{\mu}-P M_{\mu} C_{\sigma^{-1}}(1-P) C_{\sigma} P .
\end{aligned}
$$

Because $(1-P) C_{\sigma} P=\left[C_{\sigma}, P\right] P=\left[C_{\sigma}, H\right] P / 2$, which according to Theorem 2.1 is compact, $A=T_{\mu}+K$ with some $K \in \mathscr{K}$. Hence $A$ is an invertible operator in $\mathscr{T}(C)$. Since $\left(P C_{\sigma} P\right)\left(P C_{\sigma^{-1}} P\right)=1+$ compact, we have $\left(P C_{\sigma} P\right)^{-1}=P C_{\sigma^{-1}} P+L$, where $L$ is compact. Note that for any $B \in \mathscr{T}(C)$ and any $S \in \mathscr{T}\left(L^{\infty}\right), B S-S B$ is compact. Thus, for any $f \in L^{\infty}$,

$$
\begin{aligned}
V_{\sigma} T_{f} V_{\sigma}^{*} & =\left(P C_{\sigma} P\right) A^{-1 / 2} T_{f} A^{1 / 2}\left(P C_{\sigma^{-1}} P+L\right) \\
& =P C_{\sigma} P T_{f} P C_{\sigma^{-1}} P+\text { compact } \\
& =P C_{\sigma} P M_{f} P C_{\sigma^{-1}} P+\text { compact } \\
& =P C_{\sigma} M_{f} C_{\sigma^{-1}} P+\text { compact } \\
& =P M_{f \circ \sigma} P+\text { compact }=T_{f \circ \sigma}+\text { compact. }
\end{aligned}
$$

To prove Theorem 2.1, we start with the representation of $H-C_{\sigma^{-1}} H C_{\sigma}$ as an integral operator. For $g \in C^{\infty}(\mathbf{T})=C^{\infty}$, we have

$$
\begin{aligned}
\left(H C_{\sigma} g\right)\left(e^{i \theta}\right) & =\mathrm{P} . \mathrm{V} \cdot \int_{-\pi}^{\pi} \frac{g\left(\sigma\left(e^{i t}\right)\right)}{e^{i t}-e^{i \theta}} d e^{i t} / 2 \pi i \\
& =\mathrm{P} . \mathrm{V} \cdot \int_{-\pi}^{\pi} \frac{g\left(e^{i s}\right)}{\sigma^{-1}\left(e^{i s}\right)-e^{i \theta}}\left(\frac{d \sigma^{-1}\left(e^{i s}\right)}{d e^{i s}}\right) d e^{i s} / 2 \pi i .
\end{aligned}
$$

(Note that this is valid only if $\sigma$ is an orientation-preserving automorphism of T.) Hence

$$
\left(H g-C_{\sigma^{-1}} H C_{\sigma} g\right)(\tau)=\int_{\mathbf{T}} \frac{g(\gamma)}{\gamma-\tau}\left[1-\frac{\gamma-\tau}{\sigma^{-1}(\gamma)-\sigma^{-1}(\tau)} \frac{d \sigma^{-1}(\gamma)}{d \gamma}\right] d \gamma / 2 \pi i .
$$

Thus $H-C_{\sigma^{-1}} H C_{\sigma}$ has a kernel

in the sense that

$$
K_{\sigma}(\tau, \gamma)=\gamma\left[1-\frac{\gamma-\tau}{\sigma^{-1}(\gamma)-\sigma^{-1}(\tau)} \frac{d \sigma^{-1}(\gamma)}{d \gamma}\right] /(\gamma-\tau)
$$

$$
\left(H g-C_{\sigma^{-1}} H C_{\sigma} g\right)(\tau)=\int_{\mathbf{T}} K_{\sigma}(\tau, \gamma) g(\gamma) d m(\gamma) .
$$

For a homeomorphism $\sigma$ of $\mathbf{T}$ satisfying (2.1), denote

$$
\|\sigma\|=1+\left\|\frac{d \sigma}{d \tau}\right\|_{\infty}+\left\|\frac{d \sigma^{-1}}{d \tau}\right\|_{\infty} .
$$

Lemma 2.3. Let $\sigma$ be a homeomorphism of $\mathbf{T}$ satisfying (2.1), (2.2), and (2.3). Then there is an absolute constant $N$ such that the kernel $K_{\sigma}$ of $H-C_{\sigma^{-1}} H C_{\sigma}$ satisfies

$$
\left|K_{\sigma}(\tau, \gamma)\right| \leq \frac{N(1+M)\|\sigma\|^{4}}{|\tau-\gamma|^{1-\delta}},
$$

where $\delta$ and $M$ are the constants in (2.2). 
Proof. It suffices to estimate $K_{\sigma}(\sigma(\gamma), \sigma(\tau))$. Note that

$$
\begin{aligned}
K_{\sigma}(\sigma(\tau), \sigma(\gamma)) & =\gamma\left[1-\frac{\sigma(\gamma)-\sigma(\tau)}{\gamma-\tau} \frac{d \sigma^{-1}(\sigma(\gamma))}{d \sigma(\gamma)}\right] /[\sigma(\gamma)-\sigma(\tau)] \\
& =\gamma\left[\frac{\gamma-\tau}{\sigma(\gamma)-\sigma(\tau)} / \frac{d \sigma(\gamma)}{d \gamma}\right]\left[\left(\frac{d \sigma(\gamma)}{d \gamma}-\frac{\sigma(\gamma)-\sigma(\tau)}{\gamma-\tau}\right) /(\gamma-\tau)\right] .
\end{aligned}
$$

By (2.1), the lemma will follow once we show that

$$
\left|\frac{d \sigma(\gamma)}{d \gamma}-\frac{\sigma(\gamma)-\sigma(\tau)}{\gamma-\tau}\right| \leq N(1+M)\|\sigma\||\tau-\gamma|^{\delta} .
$$

It is easy to see that

$$
\begin{aligned}
& \frac{d \sigma\left(e^{i t}\right)}{d e^{i t}}-\frac{\sigma\left(e^{i t}\right)-\sigma\left(e^{i s}\right)}{e^{i t}-e^{i s}} \\
& \quad=\left[\frac{t-s}{e^{i t}-e^{i s}}\right] \frac{1}{t-s} \int_{s}^{t}\left[\frac{d \sigma\left(e^{i t}\right)}{d e^{i t}} \frac{e^{i t}-e^{i s}}{t-s}-\frac{d \sigma\left(e^{i r}\right)}{d e^{i r}} \frac{d e^{i r}}{d r}\right] d r .
\end{aligned}
$$

Thus (2.5) follows from the fact that for $r \in(s, t)$,

$$
\left|\frac{\left(e^{i t}-e^{i s}\right)}{(t-s)}-i e^{i r}\right| \leq N_{1}\left|e^{i t}-e^{i s}\right|
$$

and

$$
\left|\frac{d \sigma\left(e^{i t}\right)}{d e^{i t}}-\frac{d \sigma\left(d^{i r}\right)}{d e^{i r}}\right| \leq 2 M\left|e^{i t}-e^{i s}\right|^{\delta} .
$$

Lemma 2.4. Let $G(\tau, \gamma)$ be a nonnegative measurable function on $\mathbf{T} \times \mathbf{T}$ such that $G(\tau, \cdot) \in L^{1}(\mathbf{T})$ for a.e. $\tau \in \mathbf{T}$ and such that

$$
(G f)(\tau)=\int_{\mathbf{T}} G(\tau, \gamma) f(\gamma) d m(\gamma), \quad f \in C(\mathbf{T}),
$$

extends to a bounded operator $G$ on $L^{p}(\mathbf{T}) \quad(1<p<\infty)$ in the natural way. Then for any function $h(\cdot, \cdot) \in L^{\infty}(\mathbf{T} \times \mathbf{T})$.

$$
(K f)(\tau)=\int_{\mathbf{T}} h(\tau, \gamma) G(\tau, \gamma) f(\gamma) d m(\gamma)
$$

extends to a bounded operator on $L^{p}(\mathbf{T})$ and

$$
\|K\| \leq\|h\|_{\infty}\|G\| \text {. }
$$

Furthermore, if $G$ is compact and $h \in C(\mathbf{T} \times \mathbf{T})$, then $K$ is also compact.

Proof. Since $G(\tau, \gamma) \geq 0$, we have, for $f \in C$,

$$
|(K f)(\tau)| \leq\|h\|_{\infty} \int_{\mathbf{T}}|G(\tau, \gamma) f(\gamma)| d m(\gamma)=\|h\|_{\infty} \int_{\mathbf{T}} G(\tau, \gamma)|f(\gamma)| d m(\gamma) .
$$

Therefore

$$
\|K f\|_{p} \leq\|h\|_{\infty}\|G(|f|)\|_{p} \leq\|h\|_{\infty}\|G\|\|(|f|)\|_{p}=\|h\|_{\infty}\|G\|\|f\|_{p} .
$$

Hence $K$ extends to a bounded operator and $\|K\| \leq\|h\|_{\infty}\|G\|$. Suppose that $G$ is compact and that $h \in C(\mathbf{T} \times \mathbf{T})$. Let $\left\{h_{n}\right\}$ be a sequence of polynomials in two variables such that $\lim _{n \rightarrow \infty}\left\|h-h_{n}\right\|_{\infty}=0$. It is obvious that the kernel 
$h_{n}(\tau, \gamma) G(\tau, \gamma)$ represents a compact operator $K_{n}$. By the first part of the lemma, $\lim _{n \rightarrow 0}\left\|K-K_{n}\right\|=0$.

Lemma 2.5. Let $\delta>0$.

(i) Then

$$
\left(G_{\delta} f\right)(\tau)=\int_{\mathbf{T}}\left[\frac{f(\gamma)}{|\tau-\gamma|^{1-\delta}}\right] d m(\gamma)
$$

extends to a compact operator on $L^{2}(\mathbf{T})$. Actually $G_{\delta}$ belongs to the Schatten $q$ class for some $q>1$.

(ii) There is an $\varepsilon \in(0,1)$ such that for any $p \in(1,1+\varepsilon) \cup(1+(1 / \varepsilon), \infty)$, $G_{\delta}$ extends to a compact operator on $L^{p}(\mathbf{T})$.

Proof. Let $\varepsilon \in(0,1)$ be such that $(1-\delta)(1+\varepsilon)<1$. (i) $G_{\delta}$ is the operator of convolution with $1 /|1-\tau|^{1-\delta}$, which is a function in $L^{1+\varepsilon}(\mathbf{T})$. Therefore, with respect to the orthonormal basis $\left\{\chi_{n}: n=0,1,2, \ldots\right\}$ in $L^{2}$, where $\chi_{n}(\tau)=\tau^{n}, G_{\delta}$ is a diagonal operator. The eigenvalues of $G_{\delta}$ are precisely the Fourier coefficients of $1 /|1-\tau|^{1-\delta}$, which form an $l^{1+(1 / \varepsilon)}$ sequence [2, p. 177].

(ii) For any $p \in(1+(1 / \varepsilon), \infty), 1 /|1-\tau|^{1-\delta}$ is a function in $L^{p /(p-1)}$. Standard estimates yield that for $g \in L^{p /(p-1)}$ and $f \in L^{p},\|q * f\|_{p} \leq$ $\|g\|_{p /(p-1)}\|f\|_{p}$. Thus the operator $f \mapsto g * f$ on $L^{p}$ has a norm not greater than $\|g\|_{p /(p-1)}$. If $g$ is a polynomial then this is an operator of finite rank. For an arbitrary $g \in L^{p /(p-1)}$, the compactness of $f \mapsto g * f$ follows from the norm density of polynomials in $L^{p /(p-1)}$. Hence for any $p \in(1+(1 / \varepsilon), \infty)$, $G_{\delta}$ is compact on $L^{p}$. By the duality between $L^{p}$ and $L^{p /(p-1)}, G_{\delta}$ is also compact on $L^{p /(p-1)}$.

Lemma 2.6. Let $B_{s}, s \in[0,1]$, be Fredholm operators on a Hilbert space $\mathfrak{H}$ such that $s \mapsto B_{s}^{*} B_{s}$ and $s \mapsto B_{s} B_{s}^{*}$ are continuous functions in the operator norm topology on $[0,1]$. Then index $\left(B_{s}\right)$ is constant on $[0,1]$.

Proof. Given $s_{0} \in[0,1]$, there is an $\varepsilon>0$ such that both $\operatorname{sp}\left(B_{s_{0}}^{*} B_{s_{0}}\right) \cap(0,2 \varepsilon)$ and $\operatorname{sp}\left(B_{s_{0}} B_{s_{0}}^{*}\right) \cap(0,2 \varepsilon)$ are empty. By the continuity of $s \mapsto B_{s}^{*} B_{s}$ and $s \mapsto B_{s} B_{s}^{*}$, there is an $\alpha>0$ such that $\operatorname{sp}_{\text {ess }}\left(B_{s}^{*} B_{s}\right) \cup \operatorname{sp}_{\text {ess }}\left(B_{s} B_{s}^{*}\right) \subset(3 \varepsilon / 2, \infty)$, $\operatorname{sp}\left(B_{s}^{*} B_{s}\right) \cap[\varepsilon / 2,3 \varepsilon / 2]=\varnothing$, and $\operatorname{sp}\left(B_{s} B_{s}^{*}\right) \cap[\varepsilon / 2,3 \varepsilon / 2]=\varnothing$ whenever $\left|s-s_{0}\right| \leq$ $\alpha$. Let $\eta$ be a real-valued continuous function on $\mathbb{R}$ such that $\eta(t)=1$ for $t \leq \varepsilon / 2$ and $\eta(t)=0$ for $t \geq 3 \varepsilon / 2$. It is clear that $\eta\left(B_{s}^{*} B_{s}\right)$ and $\eta\left(B_{s} B_{s}^{*}\right)$ are projection-valued norm continuous functions for $\left|s-s_{0}\right| \leq \alpha$. Hence $\operatorname{tr}\left(\eta\left(B_{s}^{*} B_{s}\right)\right)$ and $\operatorname{tr}\left(\eta\left(B_{s} B_{s}^{*}\right)\right)$ are constants for $\left|s-s_{0}\right| \leq \alpha$. This implies that index $\left(B_{s}\right)=\operatorname{tr}\left(\eta\left(B_{s}^{*} B_{s}\right)\right)-\operatorname{tr}\left(\eta\left(B_{s} B_{s}^{*}\right)\right)$ is locally constant. Thus the constancy of index $\left(B_{s}\right)$ on $[0,1]$ follows from the connectivity of the interval.

Lemma 2.7. Let $\sigma$ be a homeomomorphism of $\mathbf{T}$ satisfying (2.1), (2.2), and (2.3). Then there is a family of homeomorphisms $\sigma_{s}, s \in[0,1]$ such that (i) each $\sigma_{s}$ satisfies (2.1) and (2.3); (ii)

$$
\left|\frac{d \sigma_{s}}{d \tau}\left(\tau_{1}\right)-\frac{d \sigma_{s}}{d \tau}\left(\tau_{2}\right)\right| \leq N(M,\|\sigma\|)\left|\tau_{1}-\tau_{2}\right|^{\delta},
$$

where $\delta$ and $M$ are the constants for $\sigma$ provided by (2.2) and $N(M,\|\sigma\|)$ is a number depending only on $M$ and $\|\sigma\|$; (iii) $\left\|\sigma_{s}\right\| \leq\|\sigma\|$ for all $s \in[0,1]$; 
(iv) $s \mapsto \sigma_{s}, s \mapsto d \sigma_{s} / d \tau$, and $s \mapsto d \sigma_{s}^{-1} / d \tau$ are norm continuous function from $[0,1]$ into $C$; and $(\mathrm{v}) \sigma_{0}(\tau)=\tau$ while $\sigma_{1}=\sigma$.

Proof. Let $\sigma\left(e^{i t}\right)=e^{i \theta(t)}$ with $-\pi \leq \theta(t) \leq \pi$. Since the lemma is obviously true if $\sigma$ is a rotation of $\mathbf{T}$, we may assume $\theta(-\pi)=-\pi$. Obviously $\theta$ is a strictly increasing function with $\theta(\pi)=\pi$. Since

$$
\frac{d \sigma\left(e^{i t}\right)}{d e^{i t}}=\frac{\left[d e^{i \theta(t)} / d t\right]}{\left[i e^{i t}\right]}=\theta^{\prime}(t)\left[\frac{\sigma\left(e^{i t}\right)}{e^{i t}}\right],
$$

$\theta^{\prime}(t)$ is a continuous, strictly positive function on $[-\pi, \pi]$ and $\theta^{\prime}(-\pi)=\theta^{\prime}(\pi)$. For each $s \in[0,1]$, let $\sigma_{s}\left(e^{i t}\right)=\exp [i s \theta(t)+i(1-s) t]$. It is easy to verify that each $\sigma_{s}$ is an orientation-preserving homeomorphism of $\mathbf{T}$ satisfying $\left\|\sigma_{s}\right\| \leq$ $\|\sigma\|$. It is also obvious that $s \mapsto \sigma_{s}, s \mapsto d \sigma_{s} / d \tau$, and $s \mapsto d \sigma_{s}^{-1} / d \tau$ are continuous. The desired estimate for $\left|d \sigma_{s}\left(\tau_{1}\right) / d \tau-d \sigma_{s}\left(\tau_{2}\right) / d \tau\right|$ follows from the comparison of $d \sigma_{s}\left(e^{i t}\right) / d e^{i t}=\left[s \theta^{\prime}(t)+(1-s)\right] \sigma_{s}\left(e^{i t}\right) / e^{i t}$ with (2.6) and the fact that $\sigma$ satisfies (2.2).

Proof of Theorem 2.1. We divide the proof into two steps. We will first show that $H-C_{\sigma^{-1}} H C_{\sigma}$ is a compact operator on $L^{2}$ and that $P C_{\sigma} P$ is a Fredholm operator of index 0 on $L^{2}$. Let $\alpha \in(1-\delta, 1)$ and $h_{\sigma}(\tau, \gamma)=K_{\sigma}(\tau, \gamma)|\tau-\gamma|^{\alpha}$, where $K_{\sigma}$ is the kernel function of $H-C_{\sigma^{-1}} H C_{\sigma}$. Since $K_{\sigma}$ satisfies (2.4) and is continuous off the diagonal of $\mathbf{T} \times \mathbf{T}, h \in C(\mathbf{T} \times \mathbf{T})$. Because $K_{\sigma}(\tau, \gamma)=h(\tau, \gamma) /|\tau-\gamma|^{\alpha}$ with $\alpha<1$, it follows from Lemmas 2.4 and 2.5 that $C_{\sigma} H-H C_{\sigma}=C_{\sigma}\left(H-C_{\sigma^{-1}} H C_{\sigma}\right)$ is a compact operator. Therefore, $(1-P) C_{\sigma} P=\left[C_{\sigma}, P\right] P=\left[C_{\sigma}, H\right] P / 2$ is compact. Hence $\left(P C_{\sigma^{-1}} P\right)\left(P C_{\sigma} P\right)=$ $P C_{\sigma^{-1}} C_{\sigma} P-P C_{\sigma^{-1}}(1-P) C_{\sigma} P=1+$ compact. Similarly $\left(P C_{\sigma} P\right)\left(P C_{\sigma^{-1}} P\right)=$ $1+$ compact. This means $P C_{\sigma} P$ is Fredholm on $H^{2}$.

To compute the index of $P C_{\sigma} P$, let us use the family of homeomorphisms $\sigma_{s}$ constructed in Lemma 2.7. We claim that $s \mapsto H-C_{\sigma_{s}^{-1}} H C_{\sigma_{s}}$ is a norm continuous function on $[0,1]$. For each $s \in[0,1]$, let $K_{\sigma_{s}}$ be the kernel of $H-C_{\sigma_{s}^{-1}} H C_{\sigma_{s}}$, and let $h_{s}(\tau, \gamma)=K_{\sigma_{s}}(\tau, \gamma)|\tau-\gamma|^{\alpha}$. By Lemma 2.4, to prove our claim it suffices to show that $s \mapsto h_{s}$ is a continuous function from $[0,1]$ into $C(\mathbf{T} \times \mathbf{T})$. By Lemmas 2.3 and 2.7 , there is a $D>0$ such that the kernel function

$$
K_{\sigma_{s}}(\tau, \gamma)=\gamma\left[1-\frac{\gamma-\tau}{\sigma_{s}^{-1}(\gamma)-\sigma_{s}^{-1}(\tau)} \frac{d \sigma_{s}^{-1}(\gamma)}{d \gamma}\right] /(\gamma-\tau)
$$

satisfies the inequality

$$
\left|K_{\sigma_{s}}(\tau, \gamma)\right| \leq \frac{D}{|\tau-\gamma|^{1-\delta}}
$$

for every $s \in[0,1]$. Hence

$$
\left|h_{s}(\tau, \gamma)\right|=\left|K_{\sigma_{s}}(\tau, \gamma)(\tau-\gamma)^{\alpha}\right| \leq D|\tau-\gamma|^{\alpha-1+\delta} .
$$

Let $s_{0} \in[0,1]$ and $\varepsilon>0$ be given. Since $\alpha-1+\delta>0$, there is a $\kappa>0$ such that $D|\tau-\gamma|^{\alpha-1+\delta}<\varepsilon / 2$ whenever $|\tau-\gamma| \leq \kappa$. Therefore, $\left|h_{s}(\tau, \gamma)-h_{t}(\tau, \gamma)\right|<$ $\varepsilon$ for any $s, t \in[0,1]$ if $|\tau-\gamma|<\kappa$. But by the construction of $\sigma_{s}$ and (2.7), there is a $\rho>0$ such that

$$
\left|h_{s_{0}}(\tau, \gamma)-h_{s}(\tau, \gamma)\right|=|\tau-\gamma|^{\alpha}\left|K_{\sigma_{s_{0}}}(\tau, \gamma)-K_{\sigma_{s}}(\tau, \gamma)\right|<\varepsilon
$$


whenever $|\tau-\gamma| \geq \kappa$ and $\left|s-s_{0}\right| \leq \rho$. This proves the continuity of $s \mapsto h_{s}$ and, therefore, that of $s \mapsto H-C_{\sigma_{s}^{-1}} H C_{\sigma_{s}}$.

Let $\mu_{s}(\tau)=d m\left(\sigma_{s}(\tau)\right) / d m(\tau)$. Then

$$
\begin{aligned}
\left(P C_{\sigma_{s}} P\right)^{*}\left(P C_{\sigma_{s}} P\right) & =P M_{\mu_{s}} C_{\sigma_{s}^{-1}} P C_{\sigma} P=T_{\mu_{s}}+P M_{\mu_{s}} C_{\sigma_{s}^{-1}}(1-P) C_{\sigma_{s}} P \\
& =T_{\mu_{s}}+P M_{\mu_{s}}\left(H-C_{\sigma_{s}^{-1}} H C_{\sigma_{s}}\right) P / 2 .
\end{aligned}
$$

Because $s \mapsto \mu_{s}$ is continuous in the norm of $C$, the function $s \mapsto$ $\left(P C_{\sigma_{s}} P\right)^{*}\left(P C_{\sigma_{s}} P\right)$ is continuous in the operator norm topology. One can draw the same conclusion for $s \mapsto\left(P C_{\sigma_{s}} P\right)\left(P C_{\sigma_{s}} P\right)^{*}$. Applying Lemmas 2.7, 2.3, and 2.5 (in that order), one concludes, by repeating the argument in the first paragraph of this proof, that every $P C_{\sigma_{s}} P$ is a Fredholm operator. According to Lemma 2.4, index $\left(P C_{\sigma_{s}} P\right)$ is constant on $[0,1]$. Since $P C_{\sigma_{0}} P=1$ on $H^{2}$, we have index $\left(P C_{\sigma} P\right)=0$.

As the second step of the proof, we will show that the kernel of $P C_{\sigma} P$ is $\{0\}$. Our basic idea is the following. A function $f \in H^{2}$ belongs to the kernel of $P C_{\sigma} P$ if and only if $f \circ \sigma \in \overline{H_{0}^{2}}$. Therefore, if we knew that $f \in L^{p}$ for every $p$, then $f, f^{2}, f^{3}, \ldots, f^{n}, \ldots$ would all belong to the kernel of $P C_{\sigma} P$. Since $P C_{\sigma} P$ is a Fredholm operator, this is possible only if $f$ is a constant. (If a function $g$ is such that the linear space spanned by $g, g^{2}, \ldots, g^{n}, \ldots$ is finite dimensional, then $g$ is a simple function. If $g$ is also analytic, then it is a constant.) Since 1 does not belong to the kernel of $P C_{\sigma} P, f$ must be 0 . Thus to complete the proof, it suffices to show that $\operatorname{ker} P C_{\sigma} P \subset \bigcap_{p<2} L^{p}$. Let $\mathfrak{H}_{1}$ and $\mathfrak{H}_{2}$ be the kernels of $P C_{\sigma} P$ and $P M_{\mu} C_{\sigma^{-1}} P\left(=\left(P C_{\sigma} P\right)^{*}\right)$, respectively. Since index $\left(P C_{\sigma} P\right)=0$, we have

$$
\operatorname{dim} \mathfrak{H}_{1}=\operatorname{dim} \mathfrak{H}_{2} .
$$

Let $0<\varepsilon<1$ be the number given in Lemma 2.5(ii). By Lemma 2.3, 2.4, 2.5, and the first part of the proof, $\left[H, C_{\sigma}\right]$ and $\left[H, C_{\sigma^{-1}}\right]$ are compact operators on $L^{p}$ for any $p \in(1,1+\varepsilon) \cup\left(1+(1 / \varepsilon, \infty)\right.$. For any $g \in C,\left[H, M_{g}\right]$ is compact on $L^{p}$. Therefore, $P M_{\mu} C_{\sigma^{-1}} P$ and $P C_{\sigma} M_{\mu^{-1}} P$ are Fredholm operators on $H^{p}$. By the first part of the proof, both $P M_{\mu} C_{\sigma^{-1}} P$ and $P C_{\sigma} M_{\mu^{-1}} P$ are Fredholm operators of index 0 on $H^{2}$. Therefore, we can choose operators

$$
K=\sum_{j=1}^{N} h_{j} \otimes k_{j} \quad \text { and } \quad L=\sum_{j=1}^{N} f_{j} \otimes g_{j}
$$

of finite rank such that $h_{j}, k_{j}, f_{j}, g_{j} \in H^{2}, j=1, \ldots, N$, and both

$$
P M_{\mu} C_{\sigma^{-1}} P+K \text { and } P C_{\sigma} M_{\mu^{-1}} P+L
$$

are invertible operators on $H^{2}$. Moreover, we may perturb $K$ and $L$ slightly without affecting the invertibility of $P M_{\mu} C_{\sigma^{-1}} P+K$ and $P C_{\sigma} M_{\mu^{-1}} P+L$. Therefore, we may assume that $h_{j}, k_{j}, f_{j}$, and $g_{j}$ all lie in $H^{2} \cap C$, so that $K$ and $L$ may also be regarded as operators on all the $H^{p}$ in the natural way.

Suppose now that $p \in(1,1+\varepsilon)$. Note that the invertibility of $P M_{\mu} C_{\sigma^{-1}} P+$ $K$ on $H^{2}$ implies that the range of $P M_{\mu} C_{\sigma^{-1}} P+K$ on $H^{p}$ contains $H^{2}$ and, therefore, is dense in $H^{p}$. Since this operator is Fredholm, we have $\left(P M_{\mu} C_{\sigma^{-1}} P+K\right) H^{p}=H^{p}$. Hence the index of $P M_{\mu} C_{\sigma^{-1}} P$ on $H^{p}$ is nonnegative. For the same reason, the index of $P C_{\sigma} M_{\mu^{-1}} P$ on $H^{p}$ is nonnegative. 
Because $\left(P M_{\mu} C_{\sigma^{-1}} P\right)\left(P C_{\sigma} M_{\mu^{-1}} P\right)=1+$ compact on $H^{p}$, we conclude that the index of $P M_{\mu} C_{\sigma^{-1}} P$ on $H^{p}$ is 0 .

The kernel of $P M_{\mu} C_{\sigma^{-1}} P$ on $H^{p}$ contains $\mathfrak{H}_{2}$ as a subspace. Therefore,

$$
\operatorname{dim}\left[H^{p} / P M_{\mu} C_{\sigma^{-1}} P H^{p}\right]=\operatorname{dim}\left[\operatorname{ker} P M_{\mu} C_{\sigma^{-1}} \mid H^{p}\right] \geq \operatorname{dim} \mathfrak{H}_{2} .
$$

Let $q=p /(p-1) \quad(\in(1+(1 / \varepsilon), \infty))$ and identify $\overline{H^{q}}$ with the dual of $H^{p}$. Then each function $\bar{g}\left(g \in H^{q}\right)$ is identified with the functional

$$
f \mapsto \int_{\mathbf{T}} f(\tau) \overline{g(\tau)} d m(\tau)
$$

on $H^{p}$. Let $\left(P M_{\mu} C_{\sigma^{-1}} P\right)^{\dagger}$ denote the operator on $\overline{H^{q}}$ dual to $P M_{\mu} C_{\sigma^{-1}} P$ on $H^{p}$. By the Hahn-Banach theorem,

$$
\operatorname{dim}\left[\operatorname{ker}\left(P M_{\mu} C_{\sigma^{-1}} P\right)^{\dagger}\right] \geq \operatorname{dim}\left[H^{p} / P M_{\mu} C_{\sigma^{-1}} P H^{p}\right] .
$$

On the other hand, it is obvious that for any $g \in H^{q}$,

$$
\left(P M_{\mu} C_{\sigma^{-1}} P\right)^{\dagger} \bar{g}=\overline{\left(P C_{\sigma} P g\right)} \text {. }
$$

Let $\mathfrak{H}_{1}^{q}$ be the kernel of $P C_{\sigma} P$ on $H^{q} \cdot(2.8)-(2.11)$ imply that $\operatorname{dim} \mathfrak{H}_{1}^{q} \geq$ $\operatorname{dim} \mathfrak{H}_{1}$. Since $q=p /(p-1)>2$, we have $\mathfrak{H}_{1}^{q} \subset \mathfrak{H}_{1}$. Hence $\mathfrak{H}_{1}^{q}=\mathfrak{H}_{1}$ for every $q \in(1+(1 / \varepsilon), \infty)$. This concludes the proof.

\section{3. $V_{\sigma}$ AS A FUNCTION OF $\sigma$}

If $\sigma$ is a homeomorphism of $\mathbf{T}$ satisfying (2.1)-(2.3), then one can ask how many different unitary operators satisfy the conclusion of theorem 2.2 ? The following proposition answers this question. In it, we let $Q C=\left[H^{\infty}+C\right] \cap$ $\overline{[H \infty+C]}$.

Proposition 3.1. Let $\sigma$ be a homeomorphism of $T$ satisfying (2.1), (2.2), and (2.3), and let $V_{\sigma}$ and $U_{\sigma}$ be unitary operators on $H^{2}$ satisfying the conclusion of Theorem 2.2. Then there is a unitary operator $W$ in $\mathscr{T}(Q C)$ such that $V_{\sigma}=W U_{\sigma}$.

Proof. This is immediate from [3] where Davidson shows that an operator $W$ on $H^{2}$ essentially commutes with $\mathscr{T}\left(L^{\infty}\right)$ if and only if $W$ lies in $\mathscr{T}(Q C)$, i.e., $W$ satisfies $\left[W, T_{\varphi}\right] \in \mathscr{K}$ for all $\varphi$ in $L^{\infty}$ if and only if $W \in \mathscr{T}(Q C)$.

Of course this raises the question: What are the operators that commute with $\mathscr{T}\left(L^{\infty}\right)$ modulo $\mathscr{C}$ ? Presumably these are more than $\mathscr{T}\left(L^{\infty}\right)$, but we do not know any examples.

One may ask for an analogue of Proposition 2.1 for $\mathscr{T}(C)$, and one is then led to study the essential commutant of $\mathscr{T}(C)$. This was given a first systematic look in [1] by Barria and Halmos. Theorem 3.2, below, may help to clarify its structure. In it we require the following notation and terminology.

On $H^{2}$, an operator $D$ is called diagonal if $D \chi_{n}=a_{n} \chi_{n}$ with $a_{n} \in \mathbb{C}$, $n=0,1,2, \ldots$. For such an operator, we write $D=\operatorname{diag}\left(a_{n}\right)$. Define $U_{t}=$ $\operatorname{diag}\left(e^{2 \pi \text { int }}\right)$ for $t \in[0,1]$. Given a bounded operator $A$ on $H^{2}$, let

$$
A_{k}=\int_{0}^{1} e^{-2 \pi i k t} U_{t} A U_{t}^{*} d t, \quad k=0, \pm 1, \pm 2, \ldots
$$


Since $t \mapsto U_{t}$ is strongly continuous, the above integral converges in the strong operator topology. It is instructive to think of the $A_{k}$ as Fourier coefficients. Because $U_{s} A_{k} U_{s}^{*}=e^{2 i k s} A_{k}$, we have

$$
A_{k}=D_{k} \times \begin{cases}T_{\chi}^{k}, & k \geq 0, \\ \left(T_{\chi}^{*}\right)^{k}, & k<0,\end{cases}
$$

where $D_{k}$ is a diagonal operator. Let $\operatorname{EssCom} \mathscr{T}(C)$ be the collection of bounded operators $A$ on $H^{2}$ that commute with $\mathscr{T}(C)$ modulo $\mathscr{K}$; i.e., EssCom $\mathscr{T}(C)=\left\{A \mid A T_{\chi}-T_{\chi} A\right.$ is compact $\}$. Evidently, EssCom $\mathscr{T}(C)$ is a $C^{*}$ - algebra, and if $\varphi$ is an automorphism of $\mathscr{T}(C)$ that preserves the symbol of the Toeplitz operators, i.e., if $\varphi\left(T_{f}\right)=T_{f}+$ compact, then $\varphi$ is implemented by conjugation with a unitary operator $U$ is $\operatorname{Ess} \operatorname{Com} \mathscr{T}(C)$. So to describe these automorphisms of $\mathscr{T}(C)$, it suffices to describe the $C^{*}$-algebra EssCom $\mathscr{T}(C)$.

Theorem 3.2. Let $A$ be a bounded operator on $H^{2}$. Then $A \in \operatorname{EssCom} \mathscr{T}(C)$ if and only if it satisfies the following conditions:

(a) Let $D_{k}=\operatorname{diag}\left(a_{n}^{k}\right)$ be the diagonal operator in (2.1). Then

$$
\lim _{n \rightarrow \infty}\left(a_{n}^{k}-a_{n+1}^{k}\right)=0
$$

for $k=0, \pm 1, \pm 2, \ldots$.

(b) $t \rightarrow U_{t}\left[A, T_{\chi}\right] U_{t}^{*}$ is continuous in the operator norm.

Proof. Suppose that $A \in \operatorname{Ess} \operatorname{Com} \mathscr{T}(C)$. Then $\left[A, T_{\chi}\right] \in \mathscr{K}$. Therefore, $t \rightarrow U_{t}\left[A, T_{\chi}\right] U_{t}^{*}$ is continuous in operator norm topology. Note that

$$
\left[B_{k}, T_{\chi}\right]=\int_{0}^{1} e^{-2 \pi(k+1) i t} U_{t}\left[B, T_{\chi}\right] U_{t}^{*} d t
$$

for any bounded operator $B$ on $H^{2}$. Since $t \rightarrow U_{t}\left[A, T_{\chi}\right] U_{t}^{*}$ is a compact operator-valued function, $\left[A_{k}, T_{\chi}\right]$ is a compact operator. It follows from (3.1) that $\left[D_{k}, T_{\chi}\right]$ is compact, which in turn implies (3.2).

Suppose now that $A$ satisfies (a) and (b). For $r \in(0,1)$, let $P_{r}(t)=1+$ $\sum_{k=1}^{\infty} r^{k}\left(e^{2 \pi i k t}+e^{-2 \pi i k t}\right)$. Then (b) implies that $C_{r}=\int_{0}^{1} P_{r}(t) U_{t}\left[A, T_{\chi}\right] U_{t}^{*} d t$ converges to $U_{0}\left[A, T_{\chi}\right] U_{0}^{*}=\left[A, T_{\chi}\right]$ in the operator norm as $r \rightarrow 1$. On the other hand, (a) implies that every $\left[A_{k}, T_{\chi}\right]$ is compact. Therefore, by (3.3), $C_{r}=\sum_{k=-\infty}^{\infty} r^{|k|}\left[A_{k-1}, T_{\chi}\right]$ is compact. Hence $\left[A, T_{\chi}\right]$ is compact.

\section{REFERENCES}

1. José Barría and P. R. Halmos, Asymptotic Toeplitz operators, Trans. Amer. Math. Soc. $\mathbf{2 7 3}$ (1982), 621-630.

2. P. Butzer and R. Nessel, Fourier analysis and approximation, Vol. 1, Academic Press, New York, 1971.

3. K. Davidson, On operators commuting with Toeplitz operators modulo the compact operators, J. Funct. Anal. 24 (1977), 291-302.

4. R. Douglas, Banach algebra techniques in operator theorem, Academic Press, New York, 1972.

Department of Mathematics, University of Iowa, Iowa City, Iowa 52242

E-mail address: PMUHLY@VAXA.WEEG.UIOWA.EDU

Department of Mathematics, State University of New York, Buffalo, New York, 